\title{
Effects of Herpes Simplex Virus Vector-Mediated Enkephalin Gene Therapy on Bladder Overactivity and Nociception
}

\author{
Hitoshi Yokoyama, ${ }^{1,2}$ Tomohiko Oguchi,, ${ }^{1,2}$ William F. Goins, ${ }^{3}$ James R. Goss, ${ }^{3,4}$ Osamu Nishizawa, ${ }^{2}$ \\ William C. de Groat, ${ }^{5}$ Darren Wolfe, ${ }^{4}$ David M. Krisky, ${ }^{4}$ Joseph C. Glorioso, ${ }^{3}$ and Naoki Yoshimura ${ }^{1,5}$
}

\begin{abstract}
We previously reported the effects of herpes simplex virus (HSV) vector-mediated enkephalin on bladder overactivity and pain. In this study, we evaluated the effects of vHPPE (E1G6-ENK), a newly engineered replication-deficient HSV vector encoding human preproenkephalin (hPPE). vHPPE or control vector was injected into the bladder wall of female rats 2 weeks prior to the following studies. A reverse-transcription PCR study showed high hPPE transgene levels in L6 dorsal root ganglia innervating the bladder in the vHPPE group. The number of freezing behaviors, which is a nociceptive reaction associated with bladder pain, was also significantly lower in the vHPPE group compared with the control group. The number of L6 spinal cord c-fospositive cells and the urinary interleukin (IL)-1 $\beta$ and IL-6 levels after resiniferatoxin (RTx) administration into the bladder of the vHPPE group were significantly lower compared with those of the control vector-injected group. In continuous cystometry, the vHPPE group showed a smaller reduction in intercontraction interval after RTx administration into the bladder. This antinociceptive effect was antagonized by naloxone hydrochloride. Thus, the HSV vector vHPPE encoding hPPE demonstrated physiological improvement in visceral pain induced by bladder irritation. Gene therapy may represent a potentially useful treatment modality for bladder hypersensitive disorders such as bladder pain syndrome/interstitial cystitis.
\end{abstract}

\section{Introduction}

A LTHOUGH MOST CHRONIC PAIN is not considered a lifethreatening disease, it drastically impairs the patients' quality of life (Elliott et al., 2003; Niv and Devor, 2004). Traditional pharmacologic therapies for severe chronic pain remain unsatisfactory in their ability to abate the pain response in many patients (Katz and Barkin, 2010). Opioids represent a frequently used treatment of chronic pain conditions, including bladder pain syndrome/interstitial cystitis (BPS/IC); however, activation of the opioid receptor that is unrelated to pain perception can result in adverse events such as constipation, nausea, sedation, respiratory depression, and urinary retention. The long-term use of opioids can also cause tolerance and dependency (Foley, 1993; Way, 1993). Opioid receptors have also been identified on peripheral sensory axon fibers (Stein et al., 1996; Coggeshall et al., 1997; Wenk and
Honda, 1999; Mousa et al., 2001). Numerous controlled studies of peripheral opioid analgesics, which were aimed to avoid many of these central side effects, have been used for pain management (Erickson, 1999; Ratner, 2001), but the outcome of these studies supporting preferred use of these drugs is controversial (Picard et al., 1997; Murphy et al., 2000; Stein et al., 2001). Therefore, successful treatment of chronic bladder pain really requires an ideal system to deliver opioids to target organ-specific primary afferent pathways to suppress activation of second-order neurons in the spinal cord without acting on the supraspinal region.

Gene therapy using herpes simplex virus (HSV) vectors represents a suitable candidate for the delivery of opioids to primary afferents, as the natural target of HSV is sensory nerve, where the viral genome persists as an episomal molecule within their neural cell bodies as a part of the normal life cycle of the virus (Burton et al., 2002). Moreover, HSV is a large

\footnotetext{
${ }^{1}$ Department of Urology, University of Pittsburgh School of Medicine, Pittsburgh, PA 15213.

${ }^{2}$ Department of Urology, Shinshu University School of Medicine, Matsumoto, 390-8621 Japan.

${ }^{3}$ Department of Microbiology and Molecular Genetics, University of Pittsburgh School of Medicine, Pittsburgh, PA 15219.

${ }^{4}$ Diamyd Inc., Pittsburgh, PA 15203.

${ }^{5}$ Department of Pharmacology, University of Pittsburgh School of Medicine, Pittsburgh, PA 15261.
} 
double-stranded DNA virus and about half of the HSV genome is not essential for propagation of the vector in culture, so that numerous viral genes can be deleted and replaced with multiple or large therapeutic transgenes. Replication-defective HSV vectors have been created by deleting certain immediate-early (IE) genes of the virus, resulting in vector displaying reduced cytotoxicity, thereby adding an extra margin of safety for clinical applications (Krisky et al., 1998b; Samaniego et al., 1998; Fink et al., 2000). Gene therapy approaches using HSV vectors encoding human preproenkephalin (hPPE), the precursor of met- and leu-enkephalin (which are endogenous opioids acting on $\delta$-opioid receptors), have been tested and shown to be effective for treating inflammatory, neuropathic, and visceral pain in various rat models (Wilson et al., 1999; Braz et al., 2001; Goss et al., 2001, 2002; Lu et al., 2007; Yang et al., 2008; Yokoyama et al., 2009). We have recently reported that the SHPE $\mathrm{HSV}$ vector encoding $\mathrm{hPPE}$, in which the ICP4 essential IE gene is deleted, effectively suppresses bladder overactivity and bladder pain behavior induced by intravesical application of capsaicin in rats (Yokoyama et al., 2009).

In a recent phase-I clinical trial for the treatment of cancerrelated pain from metastatic tumor, a newly engineered, less toxic HSV vector NP2 deleted for both the ICP4 and ICP27 gene products with a human cytomegalovirus (HCMV) IE promoter-driven hPPE gene expression cassette inserted into the two ICP4 loci showed that treatment of patients was well tolerated with no serious adverse events, and that subjects in the middle- and high-dose cohorts reported substantial pain relief, with those in the high-dose group having their pain scores drop from 8-10 down to 1-2 (Fink et al., 2011). However, it is not known whether this newly engineered NP2 vector that encodes hPPE, or a similar vector $v H P P E$ that contains an HCMV IE promoter-hPPE expression in the ICP4 loci of a replication-defective HSV vector deleted for ICP4 and ICP27, can be applied to the treatment of bladder hypersensitive disorders such as BPS/IC. Thus, in the current study, the vHPPE (E1G6-ENK) vector that is similar to the NP2 vector was injected into the bladder wall to examine the effects of vHPPE-mediated hPPE delivery on bladder overactivity and pain behavior induced by nociceptive stimuli in rats.

\section{Materials and Methods}

\section{Viral vectors}

The vHG control vector (Fig. 1) used in the studies (Srinivasan et al., 2007) is a replication-defective HSV-based vector deleted for the essential IE genes ICP4 and ICP27 with an HCMV IE promoter-driven enhanced green fluorescent protein (eGFP) gene expression cassette inserted into both ICP4 loci (Fig. 1). In addition, the promoters for two other IE genes, ICP22 and ICP47, were altered so that the sequences that enable them to be expressed as IE genes are altered; thus, these two IE gene products are no longer expressed during infection except when ICP4 and ICP27 are supplied in trans via their expression in the $7 \mathrm{~b}$ complementing cell line used to propagate the virus (Marconi et al., 1996; Krisky et al., 1998b), where they are expressed as early genes. This alternation means that infection of every cell that does not express ICP4 and ICP27 renders vHG defective in expression of four HSV IE genes, making it significantly less toxic than the previously tested HSV vector SHPE, which is defective in expression of only the ICP4 IE gene (Krisky et al., 1998b). The vector vHPPE was engineered from vHG by replacement of the eGFP expression cassette with an HCMV IE promoter-driven hPPE cassette in a manner similar to that for the construction of the vHGlyR vector (Srinivasan et al., 2007). This vHPPE isolate displays some similarity to the NP2 vector used in the phase-I human clinical trial (Fink et al., 2011). vHG and vHPPE were propagated in the $7 \mathrm{~b}$ complementing cell line that expresses ICP4 and ICP27, which is different from the cell line used to propagate the NP2 vector. A single 10-layer cell factory (Corning, Lowell, MA) infected with a stock of vHPPE (E1G6-ENK) (multiplicity of infection=0.01) in serum-free media was harvested using $0.45 \mathrm{M}$ salt treatment and clarified by centrifugation and filtration, and the vector was purified by ion-exchange chromatography. The product was further purified and concentrated, and then stored in cryovials at $-80^{\circ} \mathrm{C}$ until use. Virus was titered on $7 \mathrm{~b}$ cells, both prior to and after use by standard plaque assay (Marconi et al., 1996; Krisky et al., 1998b).

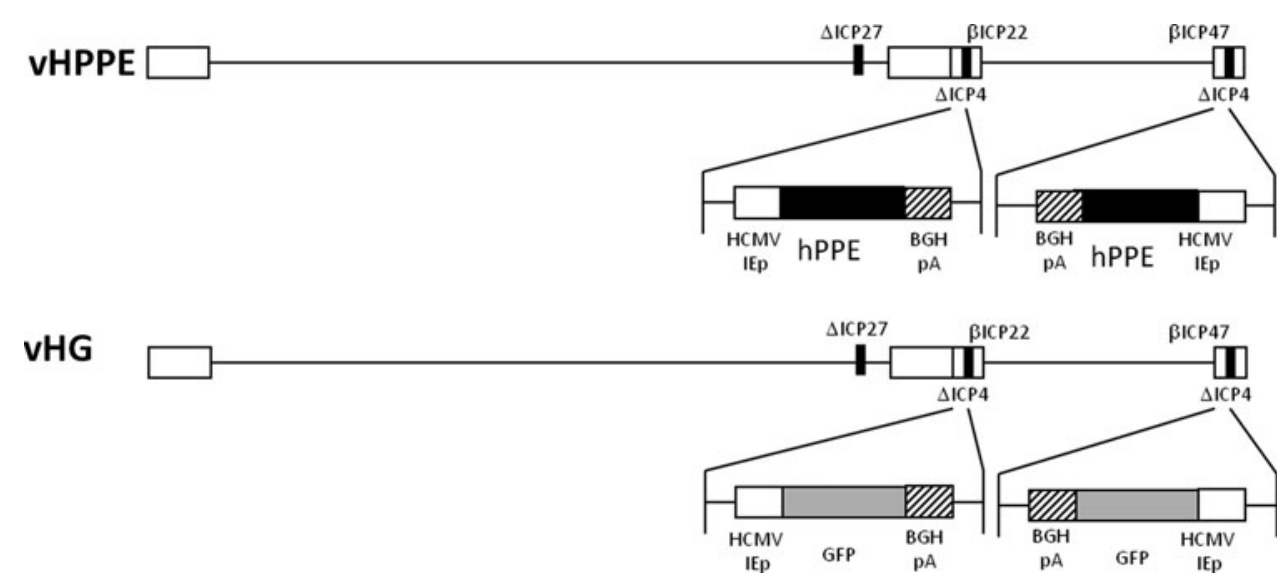

FIG. 1. Schematic representation of recombinant HSV vectors. The control vector vHG is a replication-defective HSV deleted for the ICP4 and ICP27 IE genes, in which the IE gene promoters for ICP22 and ICP47 have been mutated to allow them to be expressed as early genes only in a complementing cell line used to propagate the virus. Control vector vHG contains an HCMV promoter-eGFP expression cassette in both of the ICP4 gene loci. vHPPE is identical to vHG except that it possesses an HCMV-hPPE expression cassette in the ICP4 loci instead of eGFP. Both expression cassettes use the bovine growth hormone $(\mathrm{BGH})$ polyadenylation signal $(\mathrm{pA})$. 


\section{Viral vector administration}

All experiments were performed using female SpragueDawley rats (250-300 mg; Hilltop Animal Care, Pittsburgh, PA) in accordance with the requirements and recommendations in the Guide for the Care and the Use of Laboratory Animals (Institute for Laboratory Animal Research, 1985) approved by the University of Pittsburgh Institutional Animal Care and Use Committee. Under pentobarbital $(30 \mathrm{mg} / \mathrm{kg})$ anesthesia, a low midline incision was performed to expose the bladder, and $20 \mu \mathrm{L}$ of viral suspension [total $2.0 \times 10^{7}$ plaque-forming units (PFU)] of vHPPE or vHG control was injected at four different sites $(5 \mu \mathrm{L}$ at each point) of the anterior and posterior wall of the bladder (two sites each) using a 30-gauge Hamilton syringe (Hamilton, Reno, NV). Each site was gently pressed with a cotton swab for $30 \mathrm{sec}$ after injection to prevent leakage. The animals were treated postoperatively with antibiotics (ampicillin, $100 \mathrm{mg} / \mathrm{kg}$, intramuscularly; G.C. Hanford Manufacturing Co., Syracuse, NY). All rats were carefully monitored, and buprenorphine $[0.5 \mathrm{mg} / \mathrm{kg}$, subcutaneously (s.c.); SigmaAldrich Co., St. Louis, MO] was given to prevent pain or discomfort. The rats were housed in an approved Biosafety Level 2 animal facility.

\section{Intravesical administration of resiniferatoxin (RTx) through the urethral catheter}

RTx dissolved in 10\% ethanol, $10 \%$ Tween-80, and $80 \%$ saline was administered intravesically through the urethral catheter as described in our previous study (Saitoh et al., 2008). In brief, rats were placed in a Bollman-type restraining device (KN-326, Natsume, Tokyo, Japan). A polyethylene tube (PE-50, Clay Adams, Parsippany, NJ) was inserted into the bladder through the urethra, and residual urine was withdrawn. RTx $(3 \mu M)$ was instilled into the bladder via the catheter in a volume of $0.3 \mathrm{~mL}$ and kept for $1 \mathrm{~min}$, and then the catheter was removed from the urethra.

\section{Quantification of hPPE $m R N A$}

At 1, 2, 3, and 4 weeks after viral administration, L4 and L6 dorsal root ganglia (DRG) were removed and frozen at $-80^{\circ} \mathrm{C}$ (vHPPE, $n=5$; vHG, $\left.n=5\right)$. Total RNA was extracted by using TRIzol reagent (Invitrogen, Carlsbad, CA). One microgram of RNA was reverse-transcribed into cDNA by using SuperScript II (Invitrogen). The primers for hPPE (forward, 5' -ATTTGGGAAACCTGCAAGGA-3'; reverse, 5'GGGTGCTGGTGCCATCTT-3') and actin primer pair (Ambion, Austin, TX) were used. The hPPE and $\beta$-actin mRNA were quantified with an MX3000P real-time PCR system (Stratagene, La Jolla, CA) in a $25-\mu \mathrm{L}$ volume using SYBR Green PCR Master Mix (QIAGEN, Valencia, CA). hPPE specificity was confirmed by melting curve analysis. Sample quantification was achieved from the threshold cycle by interpolation from a standard curve to calculate a copy number for hPPE, and the ratio of hPPE to $\beta$-actin mRNA was compared.

\section{Nociceptive behavior induced by intravesical administration of $R T x$}

Two weeks after vector administration (vHPPE, $n=10$; vHG, $n=12)$, rats were placed in the metabolic cage for an acclimation for at least $2 \mathrm{hr}$. Then $3 \mu \mathrm{M}$ RTx was administered intravesically for $1 \mathrm{~min}$ through a temporary indwelling urethral catheter, and the rats were then placed back into the metabolic cage. Thereafter, licking and freezing behaviors were scored by a blinded observer over a 15-min period that was divided into 5-sec intervals. When licking or freezing occurred during each 5-sec interval, it was scored as one positive event.

\section{C-fos staining in L6 spinal cord}

Two weeks after vector administration (vHPPE, $n=5$; vHG, $n=5), 3 \mu$ M RTx was administered intravesically for $1 \mathrm{~min}$ through a temporary indwelling urethral catheter. Two hours after RTx administration, the rats were perfusion-fixed with cold heparinized saline followed by $4 \%$ paraformaldehyde (Sigma-Aldrich Co.). The L6 spinal cord was removed, postfixed overnight, and cryoprotected in $20 \%$ sucrose solution for $48 \mathrm{hr}$. The spinal cord was then cut into $40-\mu \mathrm{m}$ sections on a cryostat and incubated for $48 \mathrm{hr}$ at $4^{\circ} \mathrm{C}$ with a primary antibody (rabbit anti-c-Fos, 1:10,000; Abcam, Cambridge, MA), followed by a secondary antibody (biotinylated donkey anti-rabbit IgG, 1:600; Vector Laboratories, Burlingame, CA) for $2 \mathrm{hr}$ at room temperature, and detected with an avidin-biotin-peroxidase complex (Vecta Elite, Vector Laboratories) followed by diaminobenzidine and nickel-ammonium sulfate with hydrogen peroxide (DAB; Vector Laboratories). C-fos-positive cells were counted in four spinal cord regions of medial dorsal horn (MDH), lateral dorsal horn (LDH), dorsal commissure (DCM), and sacral parasympathetic nucleus (SPN) (Birder and de Groat, 1992) (see Fig. 4C). Control experiments ( $n=4$ each) were also conducted in rats without any procedures or with saline administration into the bladder through a urethral catheter.

\section{Cytokine/chemokine analyses in urine}

Two weeks after vector administration (vHPPE, $n=6$; vHG, $n=6), 3 \mu M$ RTx was administered intravesically through a temporary indwelling urethral catheter. Thereafter, the rats were kept in the metabolic cage, and voided urine was collected for $8 \mathrm{hr}$. Urine samples at four different points $(0-2 \mathrm{hr}, 2-4 \mathrm{hr}, 4-6 \mathrm{hr}$, and $6-8 \mathrm{hr})$ were centrifuged at $4^{\circ} \mathrm{C}$ at low speed to remove cellar debris and frozen at $-80^{\circ} \mathrm{C}$. Urinary levels of interleukin (IL)- $1 \beta$, IL-6, monocyte chemoattractant protein-1 (MCP-1), and RANTES were measured using ELISA kits (IL-6 and IL-1 $\beta$ : R\&D Systems, Minneapolis, MN; MCP-1: Thermo Scientific, Waltham, MA; RANTES: Peprotech, Rocky Hill, NJ). Urinary creatinine was also measured in each sample by the Creatinine Assay Kit (BioChain, Hayward, CA), and the levels were used to standardize urinary cytokine levels.

\section{Cystometrograms}

Two weeks after vector administration, rats were anesthetized with urethane $(1.1 \mathrm{~g} / \mathrm{kg}$, s.c.). With a lower midline abdominal incision, we exposed the bladder and inserted PE-50 tubing through the bladder dome into the bladder. Saline was then infused intravesically at $0.04 \mathrm{~mL} /$ min to induce voiding through the urethra. A software package (WinDaq, DATAQ Instruments Co., Akron, $\mathrm{OH}$ ) was used for data collection and manipulation. 
After baseline recordings were established with saline infusion, we infused RTx (10 nM) continuously into the bladder at $0.04 \mathrm{~mL} / \mathrm{min}$ to induce bladder overactivity in vHPPE $(n=8)$ and $\mathrm{vHG}(n=8)$ rats. After establishing bladder overactivity, we administered opioid receptor antagonists to delineate an opioid effect of the response to intravesical RTx. Either naloxone methiodide (Nal-M; Sigma Aldrich Co.) $(0.5 \mathrm{mg} / \mathrm{kg})(\mathrm{vHPPE}, n=4 ; \mathrm{vHG}, n=4)$, which does not pass through the blood-brain barrier (BBB), or naloxone hydrochloride (Nal-H; Sigma-Aldrich Co.) $(0.5 \mathrm{mg} / \mathrm{kg})$ (vHPPE, $n=4$; vHG, $n=4)$, which passes through the BBB, was administered intravenously.

\section{Statistics}

Nonparametric tests (Mann-Whitney U test) were used to test for differences between vHPPE and vHG intercontraction intervals (ICI) in cystometry, sensory ganglia, PPE transgene levels, and nociceptive behavior. Parametric analyses were done within groups (vHPPE and vHG) after treatments using RTx and naloxone during cystometry.

\section{Results}

\section{Quantification of the hPPE mRNA levels in L6 DRG} after $v H P P E$ injection into the bladder wall

To confirm the ability of replication-defective HSV vectors to deliver therapeutic transgenes to bladder afferent nerves after vector injection into the bladder wall, we studied vHPPE vector-mediated changes in the hPPE mRNA levels in L6 DRG, which contain bladder-innervating afferent neurons, by RT-PCR using primers specific for the hPPE cDNA that fail to hybridize to the endogenous rat PPE sequence. hPPE mRNA levels were elevated in L6 DRG of vHPPE-injected rats 2 weeks after vector injection, but not in L6 DRG from vHG-injected rats (Fig. 2A). However, in L4 DRG, which do not innervate the bladder, hPPE mRNA was not detected from vHG- or vHPPE-injected rats (Fig. 2A). The elevated hPPE mRNA levels tended to decrease during the 4-week period; however, the increased level was still detectable in L6 DRG of vHPPE-injected rats at 4 weeks after the injection (Fig. 2B).

\section{Comparison of the nociceptive behaviors induced by intravesical administration of RTx between vHPPE- and $v H G$-injected rats}

In previous studies (Craft et al., 1993; Saitoh et al., 2008; Yokoyama et al., 2009), intravesical instillation of capsaicin or RTx, a potent capsaicin analogue, induced two types of nociceptive behavior, abdominal licking (licking) and immobility, where rats point their nose toward the lower abdomen without licking (freezing). These two nociceptive behaviors were counted to evaluate bladder pain induced by intravesical application of RTx. The number of freezing behaviors during a 15-min period after intravesical administration of $3 \mu M$ RTx for 1 min was significantly reduced in vHPPE-injected rats compared with vHG-injected rats by $30 \%(46 \pm 6.2$ vs. $32 \pm 3.2, p<0.05)$ (Fig. $3 \mathrm{~A})$. When the 15-min observation period was divided into early, mid, and late 5-min segments, suppression of freezing behavior was most obvious (40\% suppression) in the late 5 -min period in vHPPE-injected rats compared with vHG-injected rats $(25 \pm 3.1$ vs. $15 \pm 2.7, p<0.05)$ (Fig. 3 C). However, there was no significant difference in RTx-induced licking behavior between vHPPE- and vHG-injected rats (74 \pm 4.4 vs. $61 \pm 3.8, p=0.080$ ) (Fig. 3B and D).

\section{Comparison of c-fos-positive cells in the L6 spinal cord after intravesical administration of RTX}

Intravesical administration of saline or RTx increased the number of c-fos-positive cells in the L6 spinal cord (Fig. 4A and B). The number of c-fos-positive cells was greater in vHG-injected rats than in vHPPE-injected rats (199 \pm 16 vs. $137 \pm 18, p<0.05)$, whereas there was no difference in the number of c-fos-positive cells after intravesical RTx infusion between vHG-injected and virus-untreated rats (Fig. 4A and B). When the L6 dorsal horn was divided into four regions consisting of MDH, LDH, DCM, and SPN (Fig 4C), the number of c-fos-positive cells was reduced in all four regions of vHPPE-injected rats, compared with vHG-injected rats, with significant differences present within the DCM $(88 \pm 4.5$ vs. $61 \pm 8.7, p<0.05)$ and SPN regions $(36 \pm 2.4$ vs. $25 \pm 2.5$, $p<0.05$ ) (Fig. 4D).
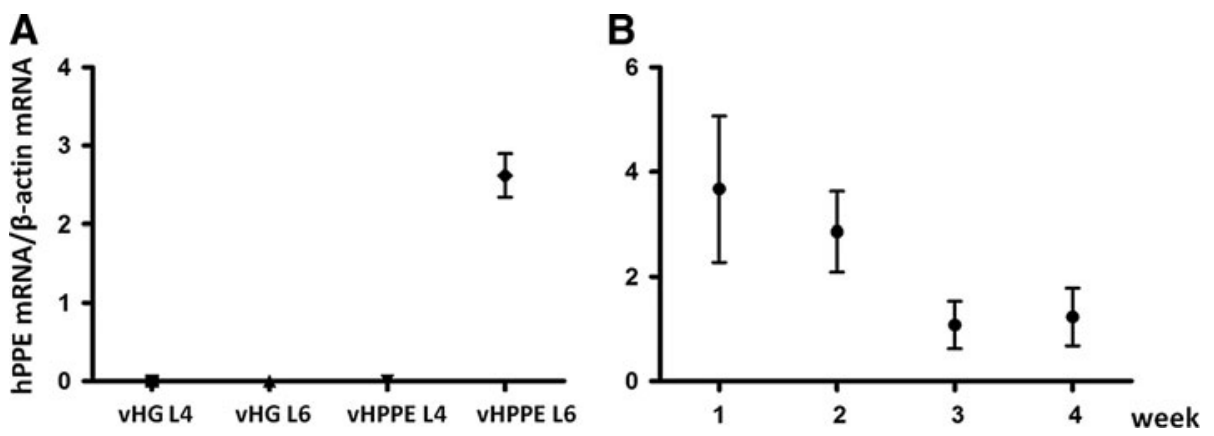

FIG. 2. RT-PCR analysis of the mRNA level of hPPE in DRG. (A) hPPE mRNA levels 2 weeks after viral injection. Either vHPPE or vHG vector was injected into the bladder wall $(n=5$ each); rats were killed at 2 weeks post injection, and tissues were isolated and used in the PCR study. hPPE mRNA was detected from L6 DRG of vHPPE-injected rats, but not in L4 and L6 DRG of vHG-injected rats and L4 DRG of vHPPE-injected rats. (B) hPPE mRNA levels from L6 DRGs of vHPPE-injected rats 1-4 weeks after viral injection. Although the levels tended to decrease during the course of the study, an increased level of hPPE mRNA was detected at 4 weeks after the injection. 
A
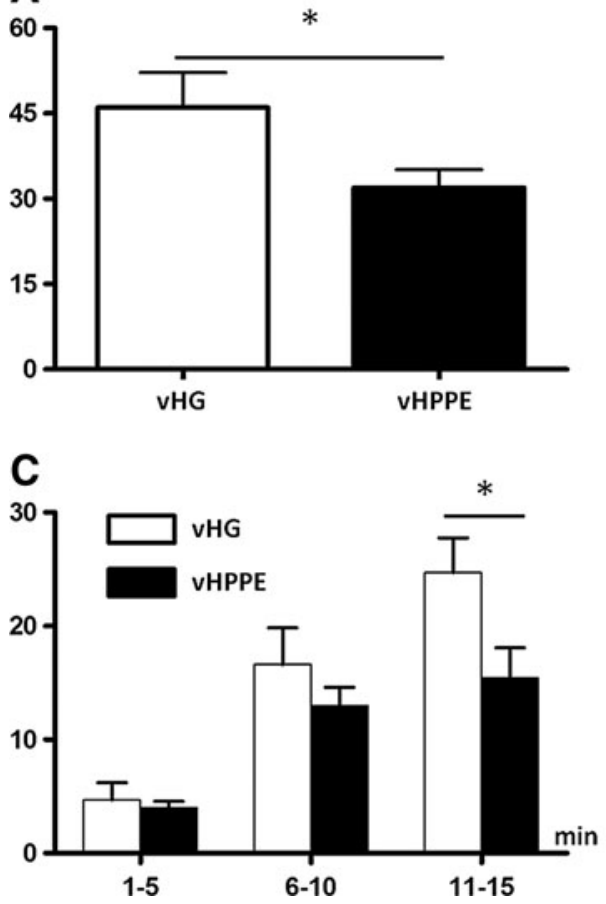

B
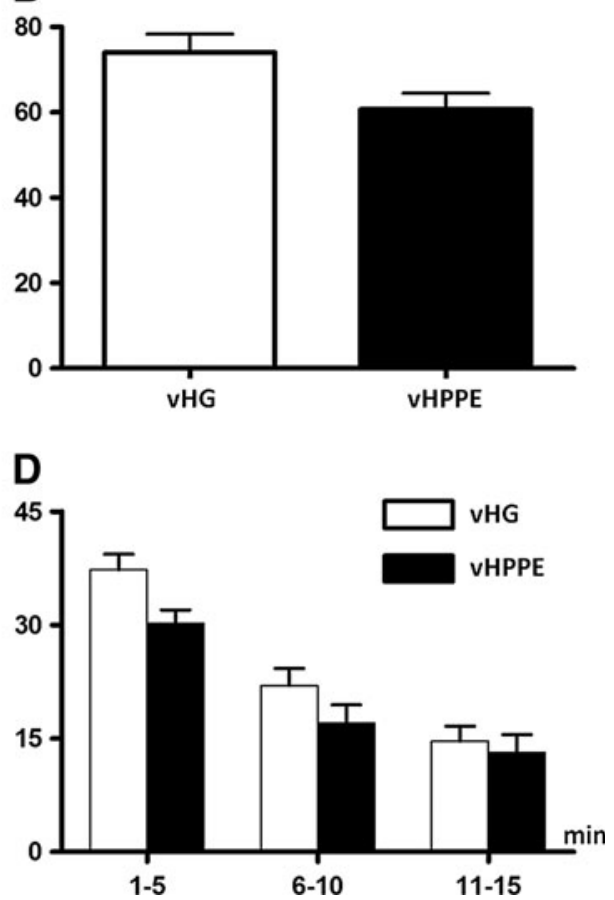

FIG. 3. Measurement of nociceptive behavior. Licking and freezing behaviors induced by intravesical application of RTx were counted every $5 \mathrm{sec}$ for $15 \mathrm{~min}$. (A) Freezing behavior in vHPPE-injected rats was suppressed significantly compared with that in vHG-treated rats. (B) Licking behavior in vHPPE-injected rats tended to decrease compared with that in vHG-treated rats, but this change did not reach significance. (C, D) Licking and freezing behaviors in the early (1-5 min), intermediate (6-10 min), and late phases (11-15 min). Late-phase freezing behavior was significantly suppressed in vHPPE-injected rats $\left({ }^{*} p<0.05\right)$. Columns and bars represent means $\pm S E M$.

\section{Comparison of urinary cytokine/chemokine levels after intravesical administration of RTx}

In vHG-injected rats, urinary levels of cytokines/chemokines such as IL-1 $\beta$, IL-6, and RANTES were significantly elevated within $4 \mathrm{hr}$ after intravesical administration of RTx, when compared with pre-RTx values, and then recovered to the baseline level within 6-8 hr (Fig. 5). However, in vHPPEinjected rats, none of these urinary cytokines/chemokines were significantly increased after intravesical RTx application compared with preapplication values, suggesting that RTx-induced increases in urine cytokines/chemokines were prevented by vHPPE treatment (Fig. 5). In addition, when the cytokine/chemokine levels were compared between two groups, the cytokine/chemokine levels after RTx administration in vHPPE-injected rats were lower compared with those in vHG-injected rats. There were significant differences between vHPPE- and vHG-injected rats in the levels of IL-1 $\beta$ (2-4 hr: $40 \pm 9.5$ vs. $180 \pm 30, p=0.0013 ; 6-8 \mathrm{hr}: 57 \pm 26$ vs. $180 \pm 25, p=0.011)$ and IL-6 (2-4hr: $41 \pm 9.8$ vs. $110 \pm 14$, $p=0.0067)$. The levels of MCP-1 and RANTES were also lower in vHPPE-injected rats compared with vHG-injected rats, although the difference did not reach statistical significance (Fig. 5).

\section{Comparison of the effect of vector-mediated enkephalin on bladder overactivity induced by intravesical administration of RTx}

During the saline infusion process, no significant differences in ICI were observed between the two groups (Fig. 6). Thereafter, when $10 \mathrm{nM}$ RTx was continuously infused into the bladder after baseline recording was established with saline infusion, both vHPPE- and vHG-injected rats showed bladder overactivity as evidenced by a significant reduction in ICI. However, the reduction of ICI in the vHPPE-injected rats was significantly smaller than that in the vHG-injected rats (ICI reduction: $39 \pm 4.9 \%$ vs. $65 \pm 5.4 \%, p=0.0035$ ) (Fig. 6 and Table 1). Administration of Nal-H significantly antagonized the inhibitory effect of vHPPE vector-mediated expression of hPPE on the ICI $(310 \pm 110 \mathrm{sec}$ pre Nal-H to $180 \pm 88 \mathrm{sec}$ post Nal-H; $p<0.05$ ) (Fig. $6 \mathrm{~B}$ and Table 2); however, Nal-M did not antagonize the inhibitory effect $(360 \pm 25 \mathrm{sec}$ pre Nal-M to $330 \pm 66 \mathrm{sec}$ post Nal-M; $p=0.53$ ) (Fig. 6A and Table 2). No change was seen in the vHGinjected rats after administration of either naloxone ( $230 \pm 48 \mathrm{sec}$ pre Nal-M to $270 \pm 50 \mathrm{sec}$ post Nal-M; $p=0.56$; $200 \pm 54 \mathrm{sec}$ pre Nal-H to $220 \pm 35 \mathrm{sec}$ post Nal-H; $p=0.73$ ) (Fig. 6C and D and Table 2).

\section{Discussion}

Various aspects of the natural biology of HSV are attractive when considering it as a gene therapy vector, especially for the treatment of diseases of the nervous system. First of all, as primary afferent neurons are the natural targets of HSV, it represents a major advantage over other vector systems, for which the target cells are not well characterized. Moreover, the vector genome is large, and about half is not essential for growth of the virus in culture for propagation; therefore, multiple (Krisky et al., 1998a) or large transgenes (Akkaraju et al., 1999) can be accommodated. Another advantage is that HSV does not integrate into the host genome (Mellerick and Fraser, 1987), so insertional mutagenesis that could potentially be tumorigenic is not a concern. Gene transfer-based delivery may also be used to provide local high levels of a gene product, while minimizing potential systemic side effects. In the current study, a genetically modified HSV vector, which is more suited for gene therapy in human patients than the previously studied HSV vector expressing hPPE, was used. Prior studies comparing early 

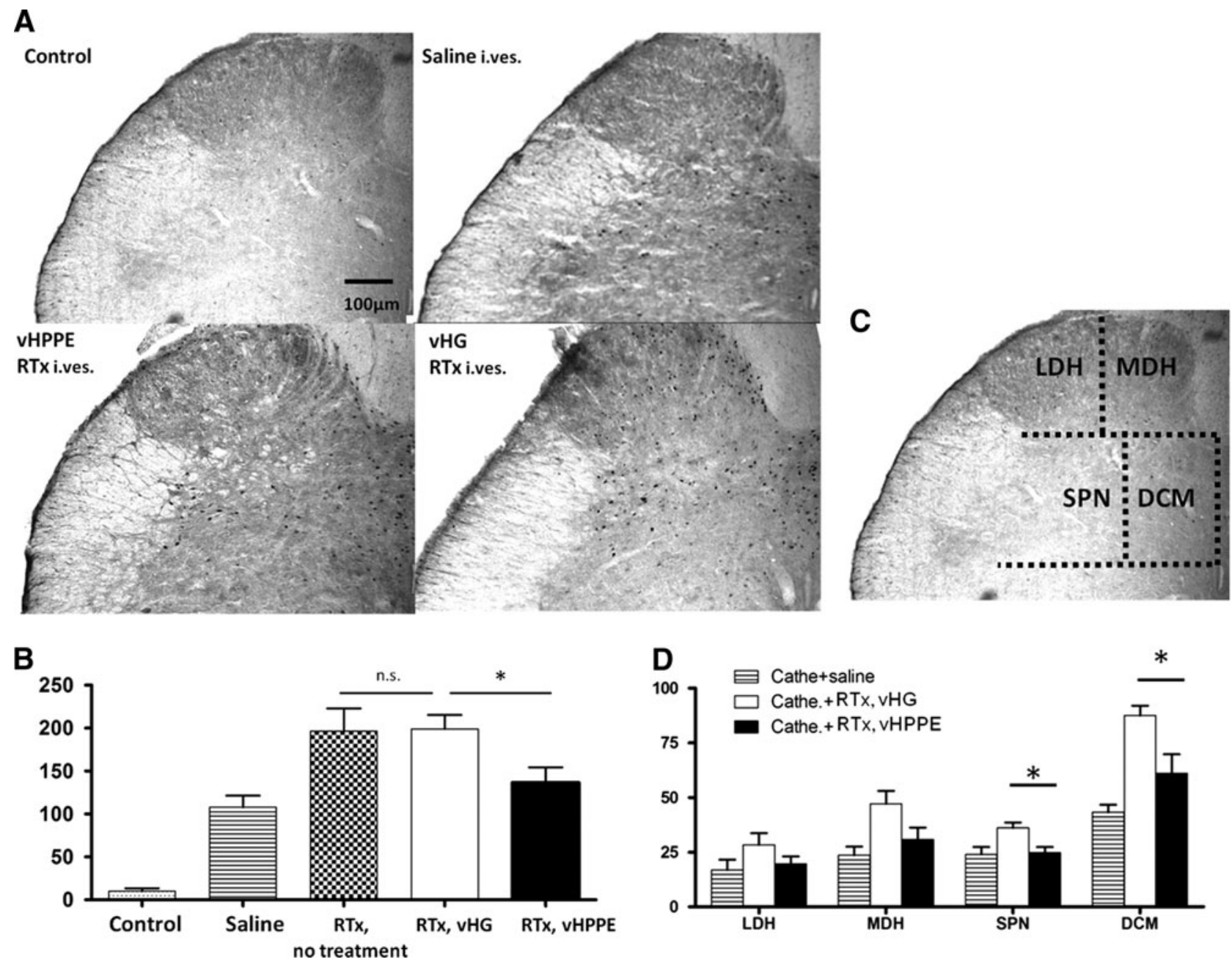

FIG. 4. C-fos staining in the spinal cord. (A) Photomicrographs of c-fos staining in the dorsal horn of L6 spinal cord of a control rat (no treatment), a saline i.ves. rat (intravesical saline injection without virus treatment), a vHPPE RTx i.ves. rat (intravesical RTx injection after vHPPE treatment), and a vHG RTx i.ves. rat (intravesical RTx injection after vHG treatment). (B) The increase of c-fos-positive cells in the L6 dorsal horn after intravesical administration of $3 \mu M$ RTx was significantly lower in vHPPE-injected rats compared with vHG-injected rats or virus-untreated rats $\left({ }^{*} p<0.05\right)$. In control rats, only a low number of c-fos-positive cells were detected. (C) A schema showing the division of the dorsal horn into four regions: lateral dorsal horn (LDH), medial dorsal horn (MDH), dorsal commissure (DCM), and sacral parasympathetic nucleus (SPN). (D) The number of c-fos-positive cells in the four regions of the L6 dorsal horn in vHPPE-injected rats was lower in all four areas compared with vHG-injected rats, with significant differences in the SPN and DCM areas $\left({ }^{*} p<0.05\right)$. Columns and bars represent means \pm SEM. n.s., not significant.

first-generation replication-defective HSV vectors deleted just for the ICP4 gene, compared with third- and fourthgeneration vectors deleted for multiple IE genes, showed that the early vectors were more toxic and expressed exceedingly lower levels of the therapeutic gene compared with those vectors deleted for multiple IE genes (Krisky et al., 1998a; Oligino et al., 1999). The new replication-defective HSV vector (Fig. 1) has the hPPE expression gene cassette inserted into the ICP4 IE gene loci under the control of the transiently active HCMV IE promoter, in the background of a vector deleted for the essential IE regulatory genes ICP4 and ICP27 containing alteration in the ICP22 and ICP47 gene promoters that prevent these genes from being expressed in normal cells, thereby rendering the new vector less cytotoxic and more amenable for human trials.
First, we confirmed transduction of the target PNS sensory neurons by the vector by measuring the hPPE presence and levels of human-specific PPE mRNA in L4 or L6 DRG (Fig. 2). These two specific DRG levels were selected because L6 DRG contain afferent neurons innervating the bladder, but L4 DRG innervate other organs and tissue. As expected, hPPE mRNA was detected only in L6 DRG, not L4 DRG (Fig. 2A), of vHPPE-injected rats for at least 4 weeks after the injection (Fig. 2B), indicating that the vector infection was limited to bladder afferent nerves and that continuous expression of enkephalin has been achieved using HSV vector gene transfer. Although we did not perform functional studies 3-4 weeks after the vector injection, our previous studies using somatic pain models reported that the antinociceptive effect of HSV vector encoding hPPE was 

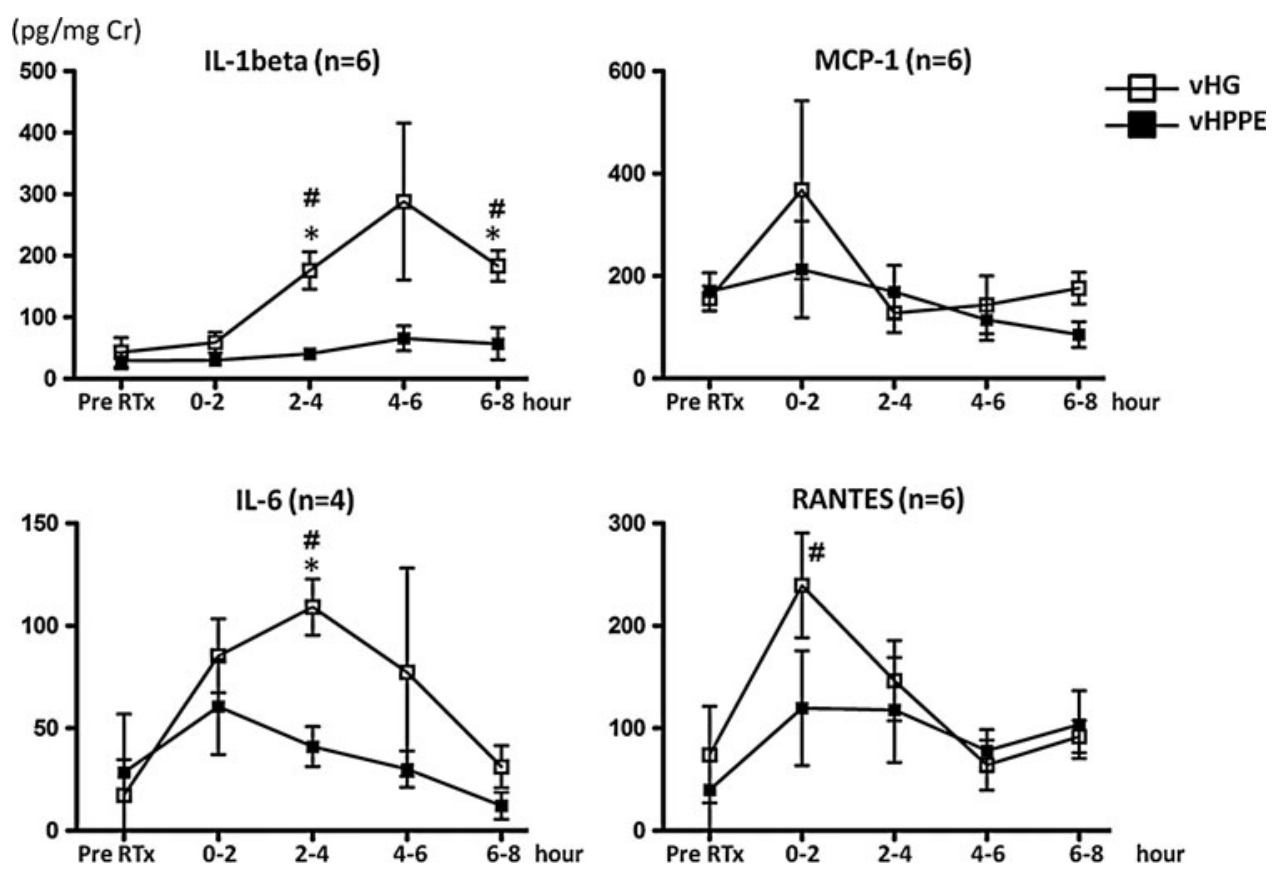

FIG. 5. Urinary cytokine/chemokine levels after intravesical administration of $3 \mu M$ RTx. In vHG-injected rats, the levels of IL-1 $\beta$, IL-6, and RANTES were significantly increased $(\# p<0.05)$ compared with pretreatment values (Pre RTx) within 4 hr and then recovered to the baseline level in 6-8 hr, whereas none of them were significantly increased after RTx administration in vHPPE-injected rats. When cytokine/chemokine levels were compared between two groups, the levels of IL-1 $\beta$ and IL-6 were significantly lower $\left({ }^{*} p<0.05\right)$ in vHPPE-injected rats when compared with vHG-injected rats. The levels of MCP-1 and RANTES tended to be lower in vHPPE-injected rats compared with vHG-injected rats; however, the difference did not reach statistical significance. Data represent means \pm SEM.

observed up to 5 weeks after virus inoculation (Hao et al., 2003), and that reinoculation of the vector reestablished the analgesic effect (Goss et al., 2001), although further studies are needed to clarify the time course of the antinociceptive effects of the vHPPE vector in rat models of cystitis. Thus, it is possible that repeated injections of HSV vectors into the bladder wall might be necessary to maintain the inhibitory effects on bladder overactivity and pain when targeting chronic bladder hypersensitive conditions such as BPS/IC.

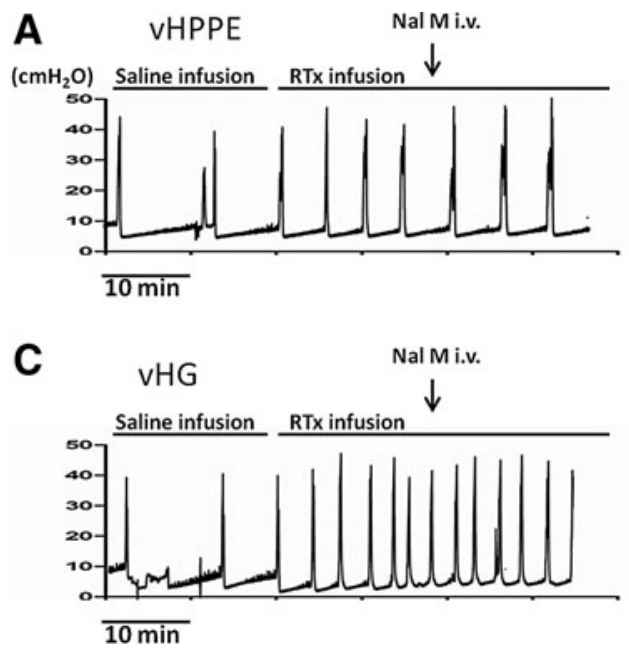

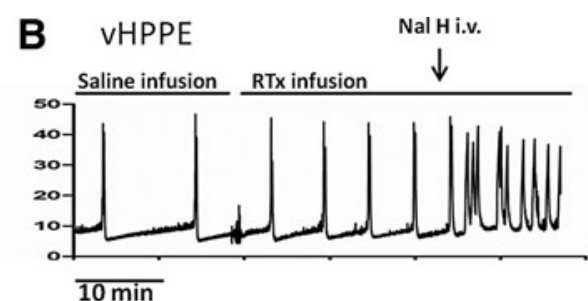

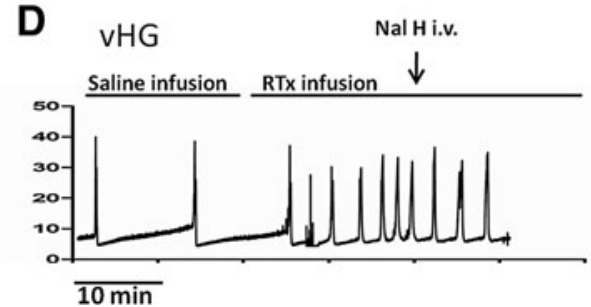

FIG. 6. Representative traces of cystometrograms in urethane-anesthetized rats. During saline infusion into the bladder, there was no difference in ICI between vHPPE-injected rats (A, B) and vHG-injected rats (C, D). After $10 \mathrm{nM}$ RTX was infused into the bladder, ICI was decreased in both vHPPE- and vHG-injected rats. However, the reduction of ICI was significantly lower in vHPPE-injected rats than in vHG-injected rats. After the RTx infusion, two types of naloxone were administered intravenously $(0.5 \mathrm{mg} / \mathrm{kg})$ : naloxone methiodide (Nal-M) and naloxone hydrochloride (Nal-H). Whereas Nal-H shortened the ICI significantly only in vHPPE-injected rats (B), Nal-M had no effect on ICI during RTx infusion into the bladder (A, C). 
TABle 1. EfFects of Intravesical Instillation of RTx ON ICI

\begin{tabular}{lcccc}
\hline Vector & $\mathrm{n}$ & $\begin{array}{c}\text { Saline } \\
\text { infusion } \\
(\mathrm{sec})\end{array}$ & $\begin{array}{c}\text { RTx } \\
\text { infusion } \\
(\mathrm{sec})\end{array}$ & $\begin{array}{c}\text { Reduction } \\
(\%)\end{array}$ \\
\hline vHPPE & 8 & $560 \pm 56$ & $340 \pm 52$ & 39 \\
vHG & 8 & $640 \pm 81$ & $220 \pm 34$ & $65^{\mathrm{a}}$ \\
\hline
\end{tabular}

${ }^{\mathrm{a}} p=0.0035$ vs. vHPPE.

Next, we investigated the effect of vHPPE expression of hPPE on bladder nociception. Intravesical administration of RTx, a capsaicin analogue, induces two types of nociceptive behavior, such as licking and freezing behavior, which we have previously seen in the rat bladder pain model (Saitoh et al., 2008). We found that RTx-induced freezing behavior was significantly suppressed in vHPPE-treated rats compared with vHG-injected rats (Fig. 3A), whereas there was no significant difference in licking behavior between these two groups (Fig. 3B). This is probably due to the fact that licking behavior is induced by stimulation of urethral afferents in the pudendal nerve rather than bladder afferents, because previous studies, including ours, have demonstrated that pudendal nerve transection significantly reduces licking behavior, but not freezing behavior, induced by intravesical application of capsaicin or RTx (Lecci et al., 1994; Saitoh et al., 2008). We have also shown that bladder wall injection of another HSV vector encoding hPPE (SHPE) suppresses freezing behavior, but not licking behavior, induced by intravesical application of capsaicin (Yokoyama et al., 2009). Thus, these results provide evidence that the effects of enkephalin gene transfer after vHPPE bladder injection are limited to bladder afferent pathways, but not the pudendal nerves, and that neither the vector nor the transgene is having pleiotropic effects on other nontarget sites. In addition, the vHPPE vector-mediated inhibitory effects in this study were smaller compared with those by SHPE vector in our previous study (Yokoyama et al., 2009). This is not in line with previous findings that early vectors, which are more toxic, expressed lower levels of the therapeutic gene compared with those vectors deleted for multiple IE genes (Krisky et al., 1998a; Goins et al., 1999; Oligino et al., 1999; Lilley et al., 2001). As the dose of SHPE vector used in the previous study was 4-40 times higher than that of the vHPPE vector used in the present study, the different dosage

TABle 2. EfFects of Intravesical Instillation OF NAL-H OR NAL-M ON ICI DURING INTRAVESICAL INSTILLATION OF RTx

\begin{tabular}{lccc}
\hline Vector & $\mathrm{n}$ & Pre Nal-H (sec) & Post Nal-H (sec) \\
\hline vHPPE & 4 & $310 \pm 110$ & $180 \pm 88^{\mathrm{a}}$ \\
vHG & 4 & $200 \pm 54$ & $210 \pm 35$ \\
\hline & & Pre Nal-M (sec) & Post Nal-M (sec) \\
\cline { 3 - 4 } vHPPE & 4 & $360 \pm 25$ & $330 \pm 66$ \\
vHG & 4 & $230 \pm 48$ & $270 \pm 50$ \\
\hline
\end{tabular}

\footnotetext{
${ }^{\mathrm{a}} p<0.05$ vs. pre Nal-H.
}

might contribute to the difference in efficacy, although further studies to compare the dose-responses of the two types of vectors are needed to clarify these points.

Then, in order to study the nociceptive input from the bladder, the number of spinal c-fos-positive cells was counted after intravesical RTx injection. Increased c-fos expression in the lumbosacral spinal cord was observed after RTx-induced bladder irritation (Fig. 4A and B). It has been reported that the most remarkable change in c-fos expression after bladder irritation occur in the DCM and then SPN regions of the L6 spinal cord in the rat (Birder and de Groat, 1992; Cruz et al., 1994, 1996; Park et al., 1997). Birder and de Groat also reported that the number of c-fos-positive cells in the DCM was related to activation of both pelvic and pudendal nerves and that the number of c-fos-positive cells in the SPN and MDH corresponds to pelvic nerve and pudendal nerve activation, respectively. In vHG-injected or virus-untreated rats, the number of c-fos-positive cells was increased more remarkably than in vHPPE-injected rats after RTx instillation into the bladder through a transurethral catheter (Fig. 4A and B). Among the four areas (LDH, MDH, SPN, and DCM) examined, significant differences were observed in the DCM and SPN regions (Fig. 4D), suggesting that bladder wall injection of $\mathrm{vHPPE}$ vectors is more likely to suppress the nociceptive response induced by activation of the pelvic nerve rather than the pudendal nerve.

There are numerous studies that demonstrate the bidirectional interaction between the nervous and immune systems. Inoue et al. reported that IL- $1 \beta$ induces substance $\mathrm{P}$ release from DRG neurons (Inoue et al., 1999). Conversely, substance $\mathrm{P}$ induced the release of IL-1 from human monocytes (Lotz et al., 1988). Although the etiology of BPS/IC is still unknown, ample studies have demonstrated that mast cell infiltration contributes at least in part to its pathogenesis, and the interaction between mast cells and cytokines/ chemokines is also proposed to have an important role in the inflammatory aspect of this disease condition. Significant changes in the urinary cytokines/chemokines were also observed in the rat model of cyclophosphamide-induced cystitis (Smaldone et al., 2009). Bouchelouche et al. suggested that after stimulation by IL- $1 \beta$ and/or tumor necrosis factor (TNF)- $\alpha$, human detrusor smooth muscle cells can produce MCP-1, which is responsible for the chemotactic migration and activation of mast cells (Bouchelouche et al., 2004). RANTES and IL-6 are also strong chemotactic molecules for mast cells (Conti et al., 1997). In addition, the expression of MCP-1 and IL- $1 \beta$ can be released by cell membrane depolarization and are up-regulated under pathological conditions in DRG neurons (Copray et al., 2001; Jung et al., 2008). Moreover, RANTES mediates TNF-dependent mast cell accumulation in the bladder lamina propria, which contributes to urothelial lesion and bladder barrier dysfunction, because neutralizing antibodies specific for RANTES blocked lamina propria mast cell accumulation, abrogated urothelial lesion, and stabilized barrier dysfunction in mice with neurogenic cystitis (Chen et al., 2007). Recently, evidence has also emerged that cytokines may be involved in the generation of pain and hyperalgesia (Sommer and Kress, 2004). IL-1 $\beta$ can act directly on sensory neurons to increase their susceptibility for noxious heat via an IL-1 receptor I/tyrosine kinase/ protein kinase C-dependent mechanism (Obreja et al., 2002). It is also known that the IL-6 level in urine is significantly 
increased or positively correlated with urinary symptoms in patients with BPS/IC (Erickson et al., 2002; Lamale et al., 2006). Taken together, these results suggest that cytokines/ chemokines such as IL-1 $\beta$, IL-6, and RANTES, the increases of which after intravesical RTx application detected in vHGinjected rats were prevented in vHPPE-injected rats in this study (Fig. 5), may represent novel therapeutic targets for the treatment of bladder inflammatory condition such as BPS/ IC. Overall, the results of the current study suggest the possibility that HSV vector-mediated enkephalin gene therapy can reduce the expression of cytokine/chemokine production in the bladder.

Lastly, we investigated the effect of vHPPE gene therapy on bladder overactivity using cystometry. During saline infusion, there was no difference in ICI between vHPPE- and vHG-injected rats. Intravesical infusion of RTx caused bladder overactivity demonstrated by a reduction of ICI in both groups (Fig. 6). However, the reduction of ICI was significantly smaller in vHPPE-injected rats compared with vHGinjected rats, indicating that vHPPE-mediated hPPE gene transfer has inhibitory effects on bladder overactivity induced by nociceptive stimuli without affecting normal micturition. As opioid receptors in the CNS including the spinal cord are known to be involved in the inhibitory control of the micturition reflex (Hisamitsu and de Groat, 1984; Dray et al., 1985), we examined if the antinociceptive effects of vHPPEmediated hPPE gene transfer is centrally mediated using $\mathrm{Nal}-\mathrm{H}$, which passes through the BBB, and Nal-M, which does not pass through the BBB. We found that Nal-H, but not Nal-M, antagonized the inhibitory effect of hPPE gene transfer on RTx-induced bladder overactivity (Fig. 6), suggesting that enkephalin released from bladder afferent nerves after vHPPE vector treatment activates opioid receptors in the spinal cord rather than those in the periphery to exert its inhibitory effects on bladder overactivity induced by bladder irritation.

We have previously reported that another HSV vector (SHPE) encoding hPPE, in which only the essential IE gene ICP4 is deleted, effectively suppresses bladder overactivity and bladder pain behavior induced by intravesical application of capsaicin in rats (Yokoyama et al., 2009). In this study, we used the newly engineered, less toxic HSV vector vHPPE deleted for the ICP4 and ICP27 loci with an HCMV IE promoter-driven hPPE gene expression cassette inserted into two ICP4 loci. A phase-I clinical study using the NP2 vector, which is similar to the vHPPE vector, has recently been performed in 10 patients who had intractable focal pain due to cancer, and showed that treatment was well tolerated with no serious adverse events and that subjects in the middleand high-dose cohorts reported substantial pain relief (Fink et al., 2011). Pain or other urinary symptoms in BPS/IC patients are known to be very difficult to treat with current therapies. Opioid drugs have been used to control pain in these patients; however, side effects are common when applied systemically (Goins et al., 2009). Thus, the targeted therapy using the HSV vector encoding hPPE, which can suppress not only bladder overactivity and pain behavior but also urine cytokine expression, could be an attractive therapeutic option without side effects, instead of current treatments including systemic opioid application. Taken together, the current study provided a foundation for the future clinical use of the newly engineered vHPPE vector in enkephalin gene transfer treatment for patients with bladder hypersensitivity disorders such as BPS/IC.

In conclusion, the current study provided the first evidence for the efficacy of gene therapy using vHPPE, a newly engineered replication-defective HSV vector encoding hPPE, for bladder pain and overactivity. The effect was limited to the bladder and bladder afferent pathways to suppress bladder cytokine production and nociceptive inputs from the bladder to the spinal dorsal horn. Moreover, this vHPPE vector, which is less cytotoxic than a previously studied one (SHPE), still has sufficient inhibitory effects on bladder nociceptive responses. Thus, gene therapy using vHPPE vectors might be a potentially useful modality for the treatment of BPS/IC.

\section{Acknowledgments}

This study was supported by National Institutes of Health grants DK57267, DK 88836, and P01 DK44935, Department of Defense grant W81XWH-12-1-0565, and The Samuel Wilan Research Fund for Interstitial Cystitis.

\section{Author Disclosure Statement}

James R. Goss, Darren Wolfe, and David M. Krisky are employees of Diamyd Inc.

\section{References}

Akkaraju, G.R., Huard, J., Hoffman, E.P., et al. (1999). Herpes simplex virus vector-mediated dystrophin gene transfer and expression in MDX mouse skeletal muscle. J. Gene Med. 1, 280-289.

Birder, L.A., and de Groat, W.C. (1992). Increased c-fos expression in spinal neurons after irritation of the lower urinary tract in the rat. J. Neurosci. 12, 4878-4889.

Bouchelouche, K., Alvarez, S., Andersen, L., et al. (2004). Monocyte chemoattractant protein-1 production by human detrusor smooth muscle cells. J. Urol. 171, 462-466.

Braz, J., Beaufour, C., Coutaux, A., et al. (2001). Therapeutic efficacy in experimental polyarthritis of viral-driven enkephalin overproduction in sensory neurons. J. Neurosci. 21, 7881-7888.

Burton, E.A., Bai, Q., Goins, W.F., and Glorioso, J.C. (2002). Replication-defective genomic herpes simplex vectors: design and production. Curr. Opin. Biotechnol. 13, 424-428.

Chen, M.C., Keshavan, P., Gregory, G.D., and Klumpp, D.J. (2007). RANTES mediates TNF-dependent lamina propria mast cell accumulation and barrier dysfunction in neurogenic cystitis. Am. J. Physiol. Renal Physiol. 292, F1372-F1379.

Coggeshall, R.E., Zhou, S., and Carlton, S.M. (1997). Opioid receptors on peripheral sensory axons. Brain Res. 764, 126-132.

Conti, P., Pang, X., Boucher, W., et al. (1997). Impact of Rantes and MCP-1 chemokines on in vivo basophilic cell recruitment in rat skin injection model and their role in modifying the protein and mRNA levels for histidine decarboxylase. Blood 89, 4120-4127.

Copray, J.C., Mantingh, I., Brouwer, N., et al. (2001). Expression of interleukin-1 beta in rat dorsal root ganglia. J. Neuroimmunol. 118, 203-211.

Craft, R.M., Carlisi, V.J., Mattia, A., et al. (1993). Behavioral characterization of the excitatory and desensitizing effects of intravesical capsaicin and resiniferatoxin in the rat. Pain 55, 205-215.

Cruz, F., Avelino, A., Lima, D., and Coimbra, A. (1994). Activation of the c-fos proto-oncogene in the spinal cord following 
noxious stimulation of the urinary bladder. Somatosens. Mot. Res. 11, 319-325.

Cruz, F., Avelino, A., and Coimbra, A. (1996). Desensitization follows excitation of bladder primary afferents by intravesical capsaicin, as shown by c-fos activation in the rat spinal cord. Pain 64, 553-557.

Dray, A., Nunan, L., and Wire, W. (1985). Central delta-opioid receptor interactions and the inhibition of reflex urinary bladder contractions in the rat. Br. J. Pharmacol. 85, 717-726.

Elliott, T.E., Renier, C.M., and Palcher, J.A. (2003). Chronic pain, depression, and quality of life: correlations and predictive value of the SF-36. Pain Med. 4, 331-339.

Erickson, D.R. (1999). Interstitial cystitis: update on etiologies and therapeutic options. J. Womens Health Gend. Based Med. 8, 745-758.

Erickson, D.R., Xie, S.X., Bhavanandan, V.P., et al. (2002). A comparison of multiple urine markers for interstitial cystitis. J. Urol. 167, 2461-2469.

Fink, D.J., DeLuca, N.A., Yamada, M., et al. (2000). Design and application of HSV vectors for neuroprotection. Gene Ther. 7, 115-119.

Fink, D.J., Wechuck, J., Mata, M., et al. (2011). Gene therapy for pain: results of a phase I clinical trial. Ann. Neurol. 70, 207-212.

Foley, K.M. (1993). Opioid analgesics in clinical pain management. In Opioid II: Handbook of Experimental Pharmacology. A. Herz, H. Akil, and E.J. Simon, eds. (Springer-Verlag, New York) pp. 697-744.

Goins, W.F., Lee, K.A., Cavalcoli, J.D., et al. (1999). Herpes simplex virus type 1 vector-mediated expression of nerve growth factor protects dorsal root ganglion neurons from peroxide toxicity. J. Virol. 73, 519-532.

Goins, W.F., Goss, J.R., Chancellor, M.B., et al. (2009). Herpes simplex virus vector-mediated gene delivery for the treatment of lower urinary tract pain. Gene Ther. 16, 558-569.

Goss, J.R., Mata, M., Goins, W.F., et al. (2001). Antinociceptive effect of a genomic herpes simplex virus-based vector expressing human proenkephalin in rat dorsal root ganglion. Gene Ther. 8, 551-556.

Goss, J.R., Harley, C.F., Mata, M., et al. (2002). Herpes vectormediated expression of proenkephalin reduces bone cancer pain. Ann. Neurol. 52, 662-665.

Hao, S., Mata, M., Goins, W., et al. (2003). Transgene-mediated enkephalin release enhances the effect of morphine and evades tolerance to produce a sustained antiallodynic effect in neuropathic pain. Pain 102, 135-142.

Hisamitsu, T., and de Groat, W.C. (1984). The inhibitory effect of opioid peptides and morphine applied intrathecally and intracerebroventricularly on the micturition reflex in the cat. Brain Res. 298, 51-65

Inoue, A., Ikoma, K., Morioka, N., et al. (1999). Interleukin-1 $\beta$ induces substance $P$ release from primary afferent neurons through the cyclooxygenase-2 system. J. Neurochem. 73, 2206-2213.

Jung, H., Toth, P.T., White, F.A., and Miller, R.J. (2008). Monocyte chemoattractant protein-1 functions as a neuromodulator in dorsal root ganglia neurons. J. Neurochem. 104, 254-263.

Katz, W.A., and Barkin, R.L. (2010). Dilemmas in chronic/ persistent pain management. Dis. Mon. 56, 233-250.

Krisky, D.M., Marconi, P.C., Oligino, T.J., et al. (1998a). Development of herpes simplex virus replication-defective multigene vectors for combination gene therapy applications. Gene Ther. 5, 1517-1530.

Krisky, D.M., Wolfe, D., Goins, W.F., et al. (1998b). Deletion of multiple immediate-early genes from herpes simplex virus reduces cytotoxicity and permits long-term gene expression in neurons. Gene Ther. 5, 1593-1603.

Lamale, L.M., Lutgendorf, S.K., Zimmerman, M.B., and Kreder, K.J. (2006). Interleukin-6, histamine, and methylhistamine as diagnostic markers for interstitial cystitis. Urology 68, 702-706. Lecci, A., Giuliani, S., Lazzeri, M., et al. (1994). The behavioral response induced by intravesical instillation of capsaicin rats is mediated by pudendal urethral sensory fibers. Life Sci. 55, 429-436.

Lilley, C.E., Groutsi, F., Han, Z., et al. (2001). Multiple immediate-early gene-deficient herpes simplex virus vectors allowing efficient gene delivery to neurons in culture and widespread gene delivery to the central nervous system in vivo. J. Virol. 75, 4343-4356.

Lotz, M., Vaughan, J.H., and Carson, D.A. (1988). Effect of neuropeptides on production of inflammatory cytokines by human monocytes. Science 241, 1218-1221.

Lu, Y., McNearney, T.A., Lin, W., et al. (2007). Treatment of inflamed pancreas with enkephalin encoding HSV-1 recombinant vector reduces inflammatory damage and behavioral sequelae. Mol. Ther. 15, 1812-1819.

Marconi, P., Krisky, D., Oligino, T., et al. (1996). Replicationdefective herpes simplex virus vectors for gene transfer in vivo. Proc. Natl. Acad. Sci. U.S.A. 93, 11319-11320.

Mellerick, D.M., and Fraser, N.W. (1987). Physical state of the latent herpes simplex virus genome in a mouse model system: evidence suggesting an episomal state. Virology 158, 265-275.

Mousa, S.A., Zhang, Q., Sitte, N., et al. (2001). $\beta$-Endorphincontaining memory-cells and $\mu$-opioid receptors undergo transport to peripheral inflamed tissue. J. Neuroimmunol. 115, 71-78. Murphy, D.B., McCartney, C.J., and Chan, V.W. (2000). Novel analgesic adjuncts for brachial plexus block: a systematic review. Anesth. Analg. 90, 1122-1128.

Niv, D., and Devor, M. (2004). Chronic pain as a disease in its own right. Pain Pract. 4, 179-181.

Obreja, O., Schmelz, M., Poole, S., and Kress, M. (2002). Interleukin6 in combination with its soluble IL-6 receptor sensitises rat skin nociceptors to heat, in vivo. Pain 96, 57-62.

Oligino, T., Ghivizzani, S., Wolfe, D., et al. (1999). Intra-articular delivery of a herpes simplex virus IL-1Ra gene vector reduces inflammation in a rabbit model of arthritis. Gene Ther. 6, 1713-1720.

Park, M., Kim, J., Bae, Y., et al. (1997). CNS innervation of the urinary bladder demonstrated by immunohistochemical study for c-fos and pseudorabies virus. J. Korean Med. Sci. 12, 340-352.

Picard, P.R., Tramer, M.R., McQuay, H.J., and Moore, R.A. (1997). Analgesic efficacy of peripheral opioids (all except intra-articular): a qualitative systematic review of randomised controlled trials. Pain 72, 309-318.

Ratner, V. (2001). Interstitial cystitis: a chronic inflammatory bladder condition. World J. Urol. 19, 157-159.

Saitoh, C., Chancellor, M.B., de Groat, W.C., and Yoshimura, N. (2008). Effects of intravesical instillation of resiniferatoxin on bladder function and nociceptive behavior in freely moving, conscious rats. J. Urol. 179, 359-364.

Samaniego, L.A., Neiderhiser, L., and DeLuca, N.A. (1998). Persistence and expression of the herpes simplex virus genome in the absence of immediate-early proteins. J. Virol. 72, 3307-3320.

Smaldone, M.C., Vodovotz, Y., Tyagi, V., et al. (2009). Multiplex analysis of urinary cytokine levels in rat model of cyclophosphamide-induced cystitis. Urology 73, 421-426.

Sommer, C., and Kress, M. (2004). Recent findings on how proinflammatory cytokines cause pain: peripheral mechanisms 
in inflammatory and neuropathic hyperalgesia. Neurosci. Lett. 361, 184-187.

Srinivasan, R., Huang, S., Chaudhry, S., et al. (2007). An HSV vector system for selection of ligand-gated ion channel modulators. Nat. Methods 4, 733-739.

Stein, C., Pfluger, M., Yassouridis, A., et al. (1996). No tolerance to peripheral morphine analgesia in presence of opioid expression in inflamed synovia. J. Clin. Invest. 98, 793-799.

Stein, C., Machelska, H., and Schafer, M. (2001). Peripheral analgesic and antiinflammatory effects of opioids. Z. Rheumatol. 60, 416-424.

Way, E.L. (1993). Opioid tolerance and physical dependence and their relationship. In Opioid II: Handbook of Experimental Pharmacology. A. Herz, H. Akil, and E.J. Simon, eds. (SpringerVerlag, New York) pp. 573-596.

Wenk, H.N., and Honda, C.N. (1999). Immunohistochemical localization of delta opioid receptors in peripheral tissues. J. Comp. Neurol. 408, 567-579.

Wilson, S.P., Yeomans, D.C., Bender, M.A., et al. (1999). Antihyperalgesic effects of infection with a preproenkephalinencoding herpes virus. Proc. Natl. Acad. Sci. U.S.A. 96, 3211-3216.
Yang, H., McNearney, T.A., Chu, R., et al. (2008). Enkephalinencoding herpes simplex virus-1 decreases inflammation and hotplate sensitivity in a chronic pancreatitis model. Mol. Pain 4, 8.

Yokoyama, H., Sasaki, K., Franks, M.E., et al. (2009). Gene therapy for bladder overactivity and nociception with herpes simplex virus vectors expressing preproenkephalin. Hum. Gene Ther. 20, 63-71.

Address correspondence to: Dr. Naoki Yoshimura Suite 700 Kaufmann Building 3471 Fifth Avenue Pittsburgh, PA 15213

E-mail: nyos@pitt.edu

Received for publication October 10, 2011;

accepted after revision November 6, 2012.

Published online: January 14, 2013. 